\title{
Mesenchymal Stem Cells - Sources and Clinical Applications
}

\author{
Hans Klingemann ${ }^{\mathrm{a}} \quad$ David Matzilevich $^{\mathrm{b}}$ James Marchand $^{\mathrm{c}}$ \\ a Division of Hematology/Oncology and Molecular Oncology Research Institute, Boston, MA, \\ b New England Cord Blood Bank, Newton, MA, \\ 'Department of Anatomy, Tufts Medical Center, Boston, MA, USA
}

\section{Key Words}

Mesenchymal stem cells - MSC - Differentiation . Plasticity

\section{Summary}

Although mesenchymal stem cells (MSC) from different tissue sources share many characteristics and generally fulfill accepted criteria for MSC (plastic adherence, certain surface marker expression, and ability to differentiate into mesenchymal tissues), we are increasingly learning that they can be distinguished at the level of cytokine production and gene expression profiles. Their ability to differentiate into different tissues including endodermal and ectodermal lineages, also varies according to tissue origin. Importantly, MSC from fetal sources can undergo more cell divisions before they reach senescence than MSC from adult tissue such as bone marrow or adipose tissue. As we learn more about the differentiation and plasticity of MSC from different sources, health care providers in the future will use them tailored to different medical indications.

\section{Introduction}

Research on mesenchymal stem cells (MSC) is expanding exponentially - the cells are relatively easy to isolate from various tissues and grow/expand under standard culture conditions. They are not immunogenic, produce a plethora of cytokines, and can easily be manipulated genetically. Most importantly, in response to chemical, hormonal, or structural

\section{Schlüsselwörter}

Mesenchymale Stammzellen · MSC · Differenzierung · Plastizität

\section{Zusammenfassung}

Obwohl aus verschiedenen Geweben stammende mesenchymale Stammzellen (MSC) viele Gemeinsamkeiten aufweisen und im Allgemeinen die für MSC akzeptierten Kriterien erfüllen (plastische Adhäsion, Expression bestimmter Oberflächenmarker sowie Fähigkeit zur Differenzierung in mesenchymale Gewebe), wird immer klarer, dass sich MSC auf der Ebene der Zytokinproduktion und Genexpressionsprofile voneinander unterscheiden. Auch ihre Fähigkeit, in verschiedene Gewebe (inklusive endodermale und ektodermale Zelllinien) zu differenzieren, ist je nach Herkunftsgewebe verschieden. Nennenswerter Weise durchlaufen MSC fötalen Ursprungs mehr Zellteilungen vor Erreichen der Seneszenz als MSC aus adulten Geweben wie Knochenmark oder Fettgewebe. Mit zunehmendem Wissen über ihre Differenzierung und Plastizität werden MSC verschiedenen Ursprungs zukünftig gezielt und auf spezifische Indikationen zugeschnitten in der medizinischen Versorgung eingesetzt werden.

stimuli, MSC can differentiate into tissues of mesodermal origin such adipocytes, chondrocytes, osteocytes but can also trans-differentiate into tissues representative of all 3 embryonic layers [1-3]. No wonder that researchers flock to these cells, especially since the name 'stem cell' is associated with it. As more and more data are published on MSC from different tissue sources, it is recognized that MSC may display some differences although they may fulfill the common definition of

\begin{tabular}{ll}
\hline KARGER & $\oplus$ 2008 S. Karger GmbH, Freiburg \\
Fax +49 7614520714 & Accessible online at: \\
$\begin{array}{l}\text { E-mail Information@Karger.de } \\
\text { www.karger.com }\end{array}$ & www.karger.com/tmh
\end{tabular}


MSC: i) plastic adherence and fibroblast-like morphology (fibroblasts though have a more symmetrical morphology); ii) multipotential and multilineage differentiation capacity; iii) expression of a typical set of surface markers such as CD73, CD90, and CD105; and iv) lack of lineage-specific markers such as CD34, CD14, CD45. To keep the name stem cell for only those cells with true pluripotency, the designation 'mesenchymal stromal cells' has been suggested [4].

Differences at the genetic level have been well described for MSC from different sources [5] as have differences in the spectrum of cytokines and chemokines these cells produce [6]. It is unresolved at this point whether MSC primarily act through local production of soluble factors and how much contact is required with target tissue. Experiments using the transwell system have suggested that their immunosuppressive effect or their ability to support cell expansion can occur (albeit to lesser degree) without direct cell contact [6, 7]. Some of the enthusiasm about the clinical potential of MSC has been fueled by the finding that MSC, irrespective of their source, do not express (or only at very low levels) MHC class I antigens and have only low expression of MHC class II antigens [6]. In addition, they lack the co-stimulatory molecules of the B7 family that are required to initiate an immune response. This unique feature allows the administration of MSC preparations across MHC barriers without concern for immunological rejection and the need for immunosuppression which makes MSC a universal stem cells source.

MSC originating from the stroma of the bone marrow (BM) were one of the first known MSC, and are also most advanced in clinical trials. For those reasons, they generally serve as the 'gold standard' against which other MSC sources are compared. The following is an attempt to highlight some of the currently known differences of MSC obtained from various tissue sources. We will focus on those MSC that are easily accessible and for which some pre-clinical and/or clinical data are available or are emerging. The second part of this review will summarize the current status of the clinical development with MSC.

\section{Placenta Mesenchymal Stem Cells and Amnion Mesenchymal Stem Cells}

Both tissues are fetal in origin and believed to represent ontogenetically earlier MSC cells, as suggested by the fact that amnion MSC (A-MSC) express the pluripotency genes Oct-4 and Nanog which have not been consistently found in more mature MSC. Only 1 study has been published that compares murine A-MSC with BM-MSC [7]. Although both cell types readily differentiate into osteogenic and chondrogeneic lineages, A-MSC were unable to achieve adipogenic differentiation. The A-MSC need to be distinguished from stem cell lines obtained from amniotic fluid although they seem to have most of the characteristics of MSC [8]. Those cells were maintained for over 250 populations doublings and retained long telomeres. The placenta is another source of early MSC. Cells obtained from mid-gestation placenta express MSC markers but they are also positive for CD34 and CD49d both of which are not consistently expressed by MSC from other sources (except for Wharton's Jelly-derived MSC) [6]. Placenta-derived MSC (PL-MSC) also express genes for hematopoietic growth factors such as LIF, SCF, and TPO and have been shown to support ex vivo expansion of hematopoietic stem cells (HSCs) [9].

\section{Cord Blood Mesenchymal Stem Cells and Umbilical Cord Mesenchymal Stem Cells}

Most investigators agree with the finding that peripheral blood, even when hematopoietic growth factors are given to the blood donor, contains no or only very low numbers of MSC. In contrast to peripheral blood, about $10-30 \%$ of umbilical cord blood collections allow to culture out MSC when the cells are plated on plastic for adherence culture [10-12]. The yield may be increased when the dish is pre-coated with fetal calf serum (FCS). It appears that it is more difficult to culture umbilical cord MSC (UC-MSC) from cryopreserved samples. It takes about to 2-4 weeks after plating for cord blood MSC (CB-MSC) to start expanding, which is much longer than seen for MSC from other sources which show growth after only a few days. Although the recovery in culture is low and some of the plated cells undergo early senescence, many CB-MSC colonies show longer survival and expansion potential compared to MSC from BM or adipose tissue (AT) [11]. The MSC that are contained in $\mathrm{CB}$ (albeit in small numbers) could conceivably account for some of the beneficial effects seen after infusion in certain medical conditions such as diabetes or some neurological disorders that have been reported as improving after infusion of cord blood $[13,14]$. When tested for their differentiation potential, it was noted that UC-MSC do not form adipocytes [12]. It was also noted that CB-MSC express less CD 90 and CD105 compared to BM- or AT-MSC, although the meaning of this remains to be defined. Considering these observations, it appears that CB-MSC have some special features that distinguish them from MSC from other sources.

During embryogenesis, embryonic blood island cells migrate from the aorto-gonado-mesonephros (AGM) region through the cord towards the placenta [15]. They are accompanied by cells from the Wharton's jelly that surround these early hematopoietic cells. Those cells express the pluripotency genes Oct-4, Nanoc, and SSEA-4, and have the ability to trans-differentiate [16, 17]. Our group has shown that, compared to BM-MSC, UC-MSC produce higher levels of the hematopoietic growth factors G-CSF ad GM-CSF as well as IL-8 and IL1 [6]. Further, using gene array, we observed that UC-MSC express a host of genes with hematopoietic activity at a much higher level than BM-MSC (table 1). This could make them 
Table 1. Microarray comparison of UC-MSC and BM-MSC, up- and down-regulated genes

\begin{tabular}{|c|c|c|}
\hline Gene description & $\begin{array}{l}\text { Gene } \\
\text { symbol }\end{array}$ & $\begin{array}{l}\text { Fold } \\
\text { change } \\
\text { UCS/BMS }\end{array}$ \\
\hline Solute carrier family 7 , member 2 & SLC7A2 & 42.63 \\
\hline Desmocollin 3 & DSC3 & 34.79 \\
\hline Aldehyde dehydrogenase 1 family, member A1 & ALDH1A1 & 34.60 \\
\hline Actin, gamma 2 , smooth muscle, enteric & ACTG2 & 30.30 \\
\hline Desmoglein 2 & DSG2 & 27.70 \\
\hline Keratin 8 & KRT8 & 22.83 \\
\hline Vascular endothelial growth factor & FLT1 & 16.19 \\
\hline Fatty acid binding protein 4 , adipocyte & FABP4 & 12.19 \\
\hline Interleukin 8 & IL8 & 9.88 \\
\hline Interleukin 1 , beta & IL1B & 9.53 \\
\hline Spondin 2, extracellular matrix protein & SPON2 & 7.91 \\
\hline Leucine rich repeat containing 17 & LRRC17 & 7.38 \\
\hline ST6GALNAC5 & ST6 & 6.95 \\
\hline Reticulon 1 & RTN1 & 6.57 \\
\hline $\begin{array}{l}\text { Thyrotropin-releasing hormone degrading } \\
\text { enzyme }\end{array}$ & TRHDE & 6.55 \\
\hline Ectonucleotide pyrophosphatase (autotaxin) & ENPP2 & -16.31 \\
\hline Filaggrin & FLG & -16.13 \\
\hline Periostin, osteoblast-specific factor & POSTN & -12.91 \\
\hline Fibronectin type III domain containing 1 & FNDC1 & -12.81 \\
\hline $\begin{array}{l}\text { Natriuretic peptide receptor } \mathrm{C} \text { / guanylate } \\
\text { cyclase } \mathrm{C}\end{array}$ & NPR3 & -12.40 \\
\hline Keratin 34 & KRT34 & -10.17 \\
\hline Phosphodiesterase 1C, calmodulin-dependent & PDE1C & -8.52 \\
\hline Cathepsin K & CTSK & -8.21 \\
\hline $\begin{array}{l}\text { Ectonucleotide pyrophosphatase / } \\
\text { phosphodiesterase } 1\end{array}$ & ENPP1 & -6.96 \\
\hline Procollagen C-endopeptidase enhancer 2 & PCOLCE2 & -6.47 \\
\hline Sarcoglycan, delta & SGCD & -6.12 \\
\hline Integrin, alpha 11 & ITGA11 & -5.97 \\
\hline Solute carrier family 7 , member 8 & SLC7A8 & -5.11 \\
\hline Chemokine-like receptor 1 & CMKLR1 & -5.04 \\
\hline Crystallin, alpha B & CRYAB & -5.01 \\
\hline Early B-cell factor 3 & EBF3 & -5.00 \\
\hline Elastin & ELN & -4.87 \\
\hline
\end{tabular}

more suitable for ex vivo expansion of HSCs. We could show in a SCID mouse model that UC-MSC supported engraftment of human cord blood cells after myeloablative conditioning [6]. Further UC-MSC provide an efficient feeder layer for the multifold expansion of different human cells. Our group recently demonstrated $>60$-fold expansion of human CB-derived natural killer (NK) cells when 'Jelly' cells were used as a feeder [18]. Since those cells can easily be transfected with target genes, it is conceivable that MSC could at one point serve as custom feeder for cells that need to be expanded, including embryonic stem cells. Conversely, BM-MSC produce more VEGF and SDF-1, and hence may be more suitable to support new vessel formation or homing to the $\mathrm{BM}$ by binding to the CRCX-4 in the BM, which is the ligand for SDF-1. The UCMSC differ from BM-MSC in that they differentiate more slowly into adipocytes and chondrocytes than BM-MSC. How- ever, they have a shorter doubling time and a greater number of passages to senescence and represent earlier stage MSC than those derived from adult fat or BM.

To further define differences between UC-MSC and BMMSC, we performed microarray analysis to compare transcription profiles of both cell populations cultured under the same conditions. Significant differences were noted in the expression profiles between the two MSC types (table 1). The greatest increase (42-fold) in expression of genes in UC-MSC versus BM-MSC was seen for the gene SLC7A2, a member of the solute carrier family 7 . This gene is a cationic amino acid transporter, also known as CAT-2A, and functions to transport arginine into the cell. Numerous physiological processes are dependent upon arginine transport into the cell, including protein synthesis, nitric oxide synthesis, and creatine and agmatine synthesis [19]. Another gene with increased expression in UC-MSC versus BM-MSC (up to 34-fold) is aldehyde dehydrogenase (ALDH1A1) that is highly enriched in HSCs and, through its ability to synthesize retinoic acid, regulates differentiation of human HSCs [20]. Two other upregulated genes, desmocollin 3 and desmoglein 2, are components of desmosomes, and genes associated with cell-cell adhesion [21]. An analysis of enrichment in Gene Ontology (GO) categories was performed to determine if these genome-wide changes in UCMSC gene expression are associated with global categories of gene function. Using GOMiner [22], we determined the following gene families to be over-represented in UC-MSC: developmental processes (system development, anatomical structure development, organ development), cell signaling processes (response to external stimulus, response to chemical stimulus, cell-cell signaling, signal transduction), cell adhesion, cell migration and chemotaxis, and immune system functions (response to wounding, immune response, inflammatory response). Overall, these enriched categories give a picture of UC-MSC as preferentially involved in developmental processes, cellular motility and immune functions.

In addition to MSC in the Wharton's jelly of the cord, the perivascular area of the cord veins contains MSC which have been termed human umbilical cord perivascular cells (HUCPVC) [23, 24]. They distinguish themselves from Wharton's jelly-derived MSC by the expression of MHC class I antigens $(75 \%)$ and are negative for the pluripotency gene Oct-4. On the other hand, they express CD146, a marker that is also found on BM-MSC and dental pulp-MSC (DP-MSC) but not on UC-MSC, and appears to be a useful marker for cells that can initiate neo-vascularization and vascular repair. Although largely hypothetical at this point, it is suggested that all tissues have MSC reservoirs localized in the perivascular niche. Baksh et al. [11] found that HUCPVC generate more adipogenic cells than BM-MSC and that osteogenic differentiation proceeds more rapidly. It needs to be confirmed though if this single observation will make HUCPVC a preferred source for orthopedic and tissue engineering indications. On the other hand, HUCPVC do not differentiate into neuronal cells. 


\section{Bone Marrow Mesenchymal Stem Cells and Dental Pulp Mesenchymal Stem Cells}

With respect to preclinical development and clinical applications, BM-MSC are furthest along, and some of the ongoing clinical trials are discussed below. Obtaining them involves a bone marrow biopsy which can be a painful and inconvenient procedure. Further, BM-MSC tend to lose their proliferative capacity with age, and there seems to be a significant decline in their ability to differentiate after age 20. The group in Heidelberg [5] compared in a gene array the differential expression of genes among various MSC sources (BM, CB, AT) and compared them with HS68 fibroblasts. Twenty-five genes were overlapping and upregulated and included fibronectin, ECM2, glypican-4, ID1, NF1B, HOXA5, and HOXB6, all involved in extracellular matrix, morphogenesis, and development. It is unclear at this point as to whether these differences at the genetic level have any significance with respect to their function as adult stem cells for regenerative medicine. However, they clearly support the notion that MSC may have identical morphology or surface receptor expression, but that significant differences exist at the gene level.

During the formation of teeth, cells from the epithelial and dental papilla interact and support the differentiation of DPMSC into odontoblasts which can form primary dentin DPMSC. First studies indicate that DP-MSC have a preponderance for differentiation into bone and neurons [25, 26]. DPMSC originate from neural crest cells and mesenchymal cells during development. Neural crest cells share the same origin as progenitor cells that form the neural tissue and have the potential to differentiate into neural cell lineages. DP-MSC have been induced to express neural markers such as nestin, and are actively being investigated in preclinical models to treat Parkinson's disease as well as related neurodegenerative diseases and spinal cord injuries. In addition, they appear to replace dead neural cells and support degenerating neural cells which may have therapeutic implications for Alzheimer's disease and stroke.

\section{Adipose Tissue Mesenchymal Stem Cells}

Fat tissue (obtained from lipoaspirates) contains a heterogeneous stromal cell population that can be separated by collagenase treatment. They are positive for expression of CD49d and negative for CD106 and STO-1 all of which are expressed on BM-MSC [27]. Conversely, AD-MSC do not express BMP-2 and dlx5 which are regulators of osteogenic genes. AD-MSC are more sensitive to osteogenic induction by 1,25-dihydroxyvitamin D3, whereas BM-MSC show a better response after treatment with dexamethasone. The relevance of these observation still needs to be defined. However, it is remarkable that AD-MSC do not differentiate well into chondrocytes. Their immunosuppressive ability seems to be similar to BM-MSC [28].

\section{Miscellaneous Sources}

MSC reside essentially in all post-natal organs and tissues, and can be recruited in case of tissue injury. Their primary location may be in the perivascular area of small vessels from which they can migrate into the circulation when needed. It may therefore not be surprising that multipotential stem cells with characteristics of MSC can be found in menstrual blood, that have been given the name 'endometrial regenerative cells' [29]. The research on these cells is early, and their differentiation and particularly trans-differentiation potential still needs to be mapped. When these cells were administered to animals which had a myocardial infarct induced, functional improvement was observed [29]. One concern however that needs to be addressed for menstrual blood cells, refers to an observation that comes from BM-MSC suggesting that MSC from individuals after a certain age have shorter telomeres and are less suitable for tissue generation [11].

\section{Clinical Applications for Mesenchymal Stem Cells}

The potential clinical indications for MSC seem to be becoming endless. The largest market is in regenerative medicine and tissue engineering of mesenchymal organs such as for inherited, traumatic, or degenerative bone, joint, or cartilage repair. There is also the field of plastic surgery for reconstruction of muscle and adipose tissue, for example for patients after mastectomy or burn injuries. The other significant area of clinical use reflects the ability of MSC to home to sites of inflammatory disease and to locally impact the inflammatory/immunemediated tissue damage with subsequent ability to support tissue healing. Studies in primates have shown that BM-MSC, after intravenous infusion, distribute to many tissues [30]. They initially are trapped in the lung - likely due to their size, but then re-circulate to other organs including the gastrointestinal tissue, bone marrow, kidney, and skin. Those studies were performed in normal healthy primates - MSC however tend to localize to tissues that undergo an inflammatory response and shifting the spectrum of local cytokines from proinflammatory to anti-inflammatory. Although it is highly suspected that MSC have to be in contact with target tissue, it is known that they can also have a paracrine effect [31].

BM-MSC are the most advanced MSC with regard to clinical development. Recognizing the immunosuppressive ability of these cells, Osiris, a biotech company based in Columbia, MD, USA, has launched 2 multicenter randomized placebo controlled phase III trials for the treatment of acute graft versus host disease (GvHD). This is a dreadful complication after stem cell transplant that can cause a skin rash, and abdominal symptoms with diarrhea and liver involvement. GvHD contributes to significant morbidity and mortality after transplant. In the 'steroid resistant' trial (study no. 280), patients become eligible for MSC treatment after they have failed second line 
therapy with steroids. A second recently opened trial will test whether BM-MSC added to steroids for upfront treatment of GvHD will improve outcome (study no. 265). Over 300 patients have received BM-MSC so far without any short- or long-term side effects. Initial compassionate release treatment for patients with GvHD strongly suggested that BM-MSC can control GvHD with confirmatory observations coming from European investigators. In a phase II study, Le Blanc et al. [32] administered BM-MSC to 55 patients with grade 2-4 GvHD. The dose of MSC administered varied with a median of $1.4 \times 10^{6} / \mathrm{kg}$. Most patients received only 1-2 infusions. Both the dose and the number of infusions were different from the Osiris-sponsored trials in which patients receive a dose of $2 \times 10^{6} / \mathrm{kg}$ twice weekly for 2 (study no. 265 ) or 4 weeks (study no. 280). The European trial - although not randomized - reported a complete response rate of $68 \%$ for children and $43 \%$ for adults, which is significantly better than what is usually seen in patients with these advanced stages of acute GvHD. Importantly, no side effects were seen. One general concern is that the immunosuppressive effect of MSC may result in a potential increase in the incidence of severe infections or recurrence of the malignancy due to a weakened graft versus leukemia effect. Most patients receiving treatment for steroid resistant disease are on additional immunosuppressive medication, and the GvHD itself contributes to their severe immune deficiency. As of now, there is no indication that infusion of MSC further increases the risk of infection or cancer recurrence. This may be because INF- $\gamma$ (released during GvHD) can actually increase MHC class II expression on MSC, rendering them antigen-presenting cells for soluble antigens [33]. In a recent pilot trial, BM-MSC from the same donor were given the day before marrow transplant. Remarkably, none of the 8 patients developed severe GvHD [34]. Renal compromise is more frequently seen in patients with advanced GvHD, and some patients seem to recover renal function better when they received MSC. This may be coincidental, but a recent paper reported that mice who were given the renal toxic drug cisplatinum recovered their renal function better and faster when MSC were injected the following day [35]. BM-MSC have also shown to have a benefit when given to patients early after myocardial infarct by improving ejection fraction and lower the incidence of arrhythmias [36]. As part of that study, it was also observed that patients demonstrated an improvement in lung function compared to patients treated in the placebo group. This observation has triggered a phase II, placebo controlled trial in patients with chronic obstructive pulmonary disease (COPD) which has features of emphysema and chronic bronchitis. Another trial in patients with type I diabetes is already underway. The rationale to administer BM-
MSC in these diseases is based on their ability to down-regulate the immune response and support tissue repair processes. With the potential to be angiogenic and/or transdifferentiate into endothelial cells, bone marrow mononuclear cells are injected intrarterially in patients with peripheral vascular disease to stimulate collateral formation in lower limbs. Building on initial observation that BM-MSC seem to have an effect in controlling diarrhea in patients with intestinal GvHD, Osiris launched a study in patients with Crohn's disease who had failed a number of prior treatments. Endpoint of this study was an improvement of the Crohn's disease activity index (CDAI) by at least 100 points. This was seen in a substantial number of patients, and a randomized trial is currently underway to confirm those promising initial observation.

In addition to the indications that make use of the anti-inflammatory and immunosuppressive ability of BM-MSC, these cells have been shown to have a role in regenerative medicine. For example, BM-MSC are implanted at the site of bone defects for spinal fusion. Another 'regenerative medicine' indication is the use of MSC in osteoarthritis. Although initial studies in the goat model suggested that BM-MSC may be able to fully rebuild a damaged meniscus, this benefit could not be confirmed in clinical trials when magnetic resonance imaging of the knee was used as a read out. However, patients who received the intra-articular injection improved symptomatically which led to the conclusion that the cells may be able to reverse the osteoarthritis-related changes that had been observed in preclinical studies. Animal studies have suggested that radiation-induced tissue injury can be mitigated when MSC are infused [37,38]. Rats given MSC recovered faster from small intestine and liver damage and had faster wound healing. BM-MSC are now being stockpiled for the US Department of Defense to treat the symptoms of acute radiation syndrome (ARS) that can occur after accidental or deliberate exposure of humans to radiation. Some initial animal studies in Parkinson disease had indicated a benefit when UCMSC were given to these rats [39]. Clinical studies have not been initiated. However, a study from Korea administered BM-MSC to patients with multiple system atrophy, a disease that is associated with varying degrees of Parkinson, autonomic dysfunction, and cerebellar ataxia [40]. Eleven patients received autologous BM-MSC and a control group of 18 patients did not. In the MSC group, progression of neurological deficits and functional improvement was noticed compared to the placebo group. The therapeutic potential of MSC seems to be very promising, but we have to wait for hard data to justify the enthusiasm. We may also have to consider whether to use MSC from different sources for different disease indications. 


\section{References}

1 Pittinger MF, Mackay AM, Beck SC, et al: Multilineage potential of adult human mesenchymal stem cells. Science 1999;284:143-47.

- Javazon EH, Neggs KJ, Flake AW: Mesenchyma stem cells: paradoxes of passaging. Exp Hematol 2004:32;414-25.

$\checkmark 3$ Da Silva ML, Chagastelles PC, Nardi NB: Mesenchymal stem cells reside in virtually all post-natal organs and tissues. J Cell Sci 2006; 119: 2204-13

4 Horowitz E, Le Blanc K, Dominici M, et al: Clarification of the nomenclature for MSC: the International Society for Cellular Therapy position statement. Cytotherapy 2005;7:393-5.

$\checkmark 5$ Wagner W, Wein F, Seckinger A, et al: Comparative characteristics of mesenchymal stem cells from human bone marrow, adipose tissue, and umbilical cord blood. Exp Hematol 2005; 33: 1402-16

6 Friedman R, Betancur M, Tuncer H, Boissel L, Klingemann H: Umbilical cord mesenchymal stem cells: adjuvants for human cell transplantation, Bio Blood Marrow Transplant 2007:13:1477-86.

7 Nadri S, Soleimani M: Comparative analysis of mesenchymal stromal cells from murine bone marrow and amniotic fluid. Cytotherapy 2007;9:729-37.

8 De Coppi P, Bartsch G, Siddiqui MM, et al: Isolation of amniotic stem cell lines with potential for therapy. Nat Biotech 2007;25:100-6.

-9 Fukuchi Y, Nakajima H, Sugiyama D, Hirose I, Kitamura T, Tsuji K: Human placenta-derived cells have mesenchymal stem/progenitor cell potential. Stem Cells 2004;22:649-58

10 Secco M, Zucconi E, Vieira NM, et al: Multipotent stem cells from umbilical cord: cord is richer than blood. Stem Cells 2008;26:146-50.

11 Baksh D, Yao R, Tuan RS: Comparison of proliferative and multilineage differentiation. Potential of human mesenchymal stem cells derived from umbilical cord and bone marrow. Stem Cells 2007;25: 1484-92.

12 Kern S, Eichler H, Stoeve J, Klueter H, Bieback K: Comparative analysis of mesenchymal stem cells from bone marrow, umbilical cord blood, or adipose tissue. Stem Cells 2006;24:1294-301.

13 Ichim TE, Solano F, Glenn E, et al: Stem cell therapy for autism. J Transl Med 2007;5:30-8.

14 Koblas T, Harman SM, Saudek F: The application of umbilical cord blood cells in the treatment of diabetes mellitus. Rev Diabet Stud 2005;2:228-34.

15 Samokhvalov IM, Samokhvalov NI, Nishikawa S: Cell tracing shows the contribution of the yolk sac to adult haematopoiesis. Nature 2007;446;1056-61.
Troyer DL, Weiss ML: Wharton's jelly-derived cells are a primitive stromal cell population. Stem Cells 2007;26:591-9, review.

17 Carlin R, Davis D, Weiss M, et al: Expression of early transcription factors Oct4, Sox 2 and Nanog by porcine umbilical cord (PUC) matrix cells. Reprod Biol Endocrinol 2006;4:8-12.

18 Boissel, L, Tuncer H, Betancur M, Wolfberg A, Klingemann H: Umbilical cord mesenchymal stem cells increase expansion of cord blood natural killer cells. Biol Blood Marrow Transplant, in press.

19 Closs EI, Bosse K-P, Habermeier A, Rotmann A: Structure and function of cationic amino acid transporters (CATs). J Membrane Bio 2006;213:67-77.

20 Chute JP, Muramoto GG, Whitesides J, et al: Inhibition of aldehyde dehydrogenase and retinoid signaling induces the expansion of human hematopoietic stem cells. PNAS 2006;103:11707-12.

21 Bonne S, Gilbert B, Hatzfeld M, Chen X, Green KJ, van Roy F: Defining desmosomal plaophilin-3 interactions. J Cell Biology 2008;161:403-16.

22 Zeeberg BR, Feng W, Wang G, et al: GoMiner: a resource for biological interpretation of genomic and proteomic data. Genome Biol 2003;4:R28.

23 Karahuseyinoglu S, Cinar O, Kilic E: Biology of the stem cell in human umbilical cord stroma: in situ and in vitro surveys. Stem Cells 2006;25:319-31.

24 Sarugasser R, Lickorish D, Baksh D, Hosseini MM, Davies JE: Human umbilical cord perivascular (HUCPV) cells: a source of mesenchymal progenitors. Stem Cells 2005;23:220-9.

25 Gronthos S, Brahim J, Li W, et al: Stem cell properties of human dental pulp stem cells. J Dent Res 2002;8:531-5.

26 Yu J, Wang Y, Deng Z, Li Y, Shi J, Jin Y: Odontogenic capability: bone marrow stromal stem cells versus dental pulp stem cells. Biol Cell 2007;8: 465-74.

27 Gimble JM, Guilak F: Adipose-derived adult stem cells: isolation, characterization, and differentiation potential. Cytotherapy 2003;5:362-9.

28 Keyser KA, Beagles KE, Kiem H-P: Comparison of mesenchymal stem cells from different tissues to suppress T-cell activation. Cell Transplant 2007;16: 555-62.

29 Hida N, Nishiyama N, Miyoshi S: Novel cardiac precursor-like cells from human menstrual blood derived mesenchymal cells. Stem Cells 2008 [Epub ahead of print], Doi 10,1634/stemcells.2007-0826.
30 Devine SM, Cobbs C, Jenings M, Bartholomew A Hoffman R: Mesenchymal stem cells distribute to a wide range of tissues following systemic infusion into non-human primates. Blood 2003;101: 2999-3001.

31 Tang YL, Zhao Q, Quin X, et al: Paracrine action enhances the effects of autologous mesenchymal stem cell transplantation on vascular regeneration in rat model of myocardial infarction. Ann Thorac Surg 2005;80:229-37.

32 LeBlanc K, Frassoni F, Ball L, et al: Mesenchymal stem cells for treatment of steroid-resistant, severe, acute graft-versus-host disease: a phase II study. Lancet 2008;371:1579-86.

33 Stagg J, Pommey, S, Eliopoulos N, Galipeau J: Interferon-stimulated marrow stromal cells: a new type of non-hematopoietic antigen-presenting cell. Blood 2006;107:2570-77.

34 Alfanasyev B, Polyntsev D, Zubarovskaya L, et al: Prophylaxis and treatment of GvHD with mesenchymal stem cells in patients undergoing allo HSCT. Cytotherapy 2008;10(suppl 1):abstr 67.

35 Eliopoulos N, Zhao J, Forner K, Galipeau J: Bonemarrow derived mesenchymal stromal cells protect against cisplatin-induced acute renal failure. Cytotherapy 2008;10(suppl 1):abstr 52.

36 Luciano C, Amado LC, Saliaris AP, et al: Cardiac repair with intramyocardial injection of allogeneic mesenchymal stem cells after myocardial infarction. PNAS 2005;102:11474-9.

37 Francoise S, Bensidhoum M, Mouiseddine M, et al Local radiation not only induces homing of human mesenchymal cells at exposed sites but promotes their widespread engraftment to multiple organs: a study of their quantitative distribution after radiation damage. Stem Cells 2006;24:1020-9.

38 Chapel A, Semont A, Mouiseddine M, Gourmelon P: Human mesenchymal stem cells accelerate the healing of skin and increase intestinal recovery after radiation injury. Cytotherapy 2008;10(suppl 1):abstr 35.

39 Weiss M, Medicetty S, Bledsoe AR, et al: Human umbilical cord matrix stem cells: preliminary characterization and effect of transplantation in a rodent model of Parkinson's disease. Stem Cells 2006; 24:781-92.

40 Lee PH, Kim JW, Bang OY, Ahn YH, Joo IS, Huh $\mathrm{K}$ : Autologous mesenchymal stem cell therapy delays the progression of neurological deficits in patients with multiple system atrophy. Clin Pharmacol Therap 2008;83:723-30. 